\title{
A theorem on the absence of phase transitions in one-dimensional growth models with on-site periodic potentials
}

\author{
José A Cuesta and Angel Sánchez \\ Grupo Interdisciplinar de Sistemas Complicados (GISC), Departamento de Matemáticas, \\ Universidad Carlos III de Madrid, 28911 Leganés, Madrid, Spain \\ E-mail: cuesta@math.uc3m.es and anxo@math.uc3m.es
}

\begin{abstract}
We rigorously prove that a wide class of one-dimensional growth models with on-site periodic potential, such as the discrete sine-Gordon model, have no phase transition at any temperature $T>0$. The proof relies on the spectral analysis of the transfer operator associated with the models. We show that this operator is Hilbert-Schmidt and that its maximum eigenvalue is an analytical function of temperature.
\end{abstract}

PACS numbers: $05.70 . \mathrm{Fh}, 02.30 . \mathrm{Sa}$, 68.35.Ct, 81.10.Aj

Mathematics Subject Classification: 82B26, 46N55, 47N55, 47G10

\section{Introduction}

Physicists educated during the last half century have invariably been taught that onedimensional systems with short range forces can never have a phase transition. In point of fact, there does not exist a general proof of such a theorem (Lieb and Mattis 1966), and most instances of this statement refer to a theorem proved by van Hove (1950) that applies to a very specific class of models. Indeed, van Hove's result holds only for homogeneous fluid-like models, with pairwise interactions with a hard core and a cut-off, and in the absence of an external field. The same is true for Ruelle's extension of van Hove's theorem to lattice fluids (Ruelle 1989). In particular, external fields can give rise to phase transitions in onedimensional models, as has been recently shown (Dauxois and Peyrard 1995, Dauxois et al 2001).

In this paper, we prove that a wide class of one-dimensional models (specified below) subjected to external fields cannot exhibit phase transitions. As far as we know, this is a completely new result in so far as van Hove's theorem does not apply because, (a) there is an external field, and (b) interaction depends on degrees of freedom other than the distance 
between 'particles'. The models we discuss are especially relevant to thin film growth, as they include, as a particular case, a paradigmatic example in this field, namely the sine-Gordon model. Once again, it is very often claimed that one-dimensional 'surfaces' are always rough, but none of the many times this claim is found in the literature is supported by rigorous results; hence the importance of identifying a class of models for which this statement can be proven, as its applicability in general is, at best, dubious (Cuesta et al 2001). The work we report in this paper contributes to the advance along this direction by identifying precisely such a family of models and subsequently proving a theorem that guarantees the analyticity of the free energy. The consequences of this result and related comments close the paper.

\section{Model}

The result reported in this paper is motivated by work on the discrete sine-Gordon (sG) model which, in one dimension, is defined by

$$
\mathcal{H}_{\mathrm{sG}}=\sum_{i=1}^{N}\left\{\left(h_{i-1}-h_{i}\right)^{2}+V_{0}\left[1-\cos \left(2 \pi h_{i}\right)\right]\right\} .
$$

This model was proposed to model surface growth in two dimensions ( $h_{i}$ is understood as the height above site $i$ on a lattice) and shown to have a so-called roughening transition (the surface width becomes infinite above some finite temperature) by Chui and Weeks (1978); see also Weeks and Gilmer (1979). The rationale for such a proposal is the inclusion of the minimal ingredients intervening in the growth process: surface tension (represented by the harmonic interaction) and finite size of the atoms joining the surface (represented by the cosine term that favours integer values for the height). In one dimension, this model has been studied in detail in the late 1970s by means of a transfer operator approach (Gupta and Sutherland 1976, Currie et al 1977, Guyer and Miller 1978, Schneider and Stoll 1980); see Tsuzuki and Sasaki (1988) for a review. However, in spite of the fact that the transfer operator calculations are formally exact, the final result is in every case an expression of the partition function (and hence the free energy) in terms of the maximum eigenvalue, which cannot be computed exactly. In addition, most of these studies were concerned with the continuum limit of the sG model, i.e. the limit of the Hamiltonian (1) when the lattice spacing tends to zero. In this limit, approximate results can be obtained for the statistical mechanics of the system which suggest (e.g. Schneider and Stoll 1980) that there are no phase transitions (specifically, that the width is infinite at any non-zero temperature), but by no means constitute a rigorous proof.

In view of the lack of exact results on the existence of phase transitions for the $1 \mathrm{D} s \mathrm{~s}$ model, we set out to establish a theorem for a class of models (including the sG model) as wide as possible. To this end, we consider the systems defined by the Hamiltonian

$$
\mathcal{H}=\sum_{i=1}^{N}\left\{W\left(h_{i-1}-h_{i}\right)+B\left(h_{i}\right)\right\}
$$

where $W(x)=W(-x)$ and $B(x)=B(x+1)$ is the external field acting on every site (the periodicity being related to the lattice crystalline potential). The choice $W(x)=x^{2}$ and $B(x)=V_{0}(1-\cos 2 \pi x)$ corresponds to the discrete $\mathrm{sG}$ model (1), but our theorem includes much more general choices, as long as $W$ increases with $|x|$ sufficiently rapidly (see equation (4) and $B$ remains bounded and periodic. To fix the energy scale we may take, without loss of generality, $W(0)=B(0)=0$.

We assume periodic boundary conditions, i.e. $h_{0}=h_{N}$, and in principle the $h_{i}$ can take any real value (heights can be anything above or below an absolute reference height). However, for 
any configuration of the heights $\left\{h_{1}, \ldots, h_{N}\right\}$ there are countably many other configurations with the same energy, namely $\left\{h_{1}+k, \ldots, h_{N}+k\right\}, k \in \mathbb{Z}$, so all of them are equivalent and we can restrict our configuration space to be $\mathbb{R}^{N}$ modulo this equivalence. This is easily achieved by restricting the set of variables to be $\left(-\frac{1}{2}, \frac{1}{2}\right] \times \mathbb{R}^{N-1}$. Having specified precisely the scope of our study, we can now proceed to prove our main result.

\section{Analyticity of the free energy as a function of temperature}

The partition function for the Hamiltonian (2) is

$$
\mathcal{Z}_{N}(\beta)=\int_{-1 / 2}^{1 / 2} \mathrm{~d} h_{1} \int_{-\infty}^{\infty} \mathrm{d} h_{2} \cdots \int_{-\infty}^{\infty} \mathrm{d} h_{N} \mathrm{e}^{-\beta \mathcal{H}}
$$

where $\beta$ is the inverse of temperature in units of the Boltzmann constant. The existence of the partition function is guaranteed if $W(x)$ is such that

$$
\int_{0}^{\infty} \mathrm{d} x \mathrm{e}^{-\beta W(x)}<0 \quad \text { for all } \beta>0 .
$$

This defines the class of functions $W(x)$ for which the result holds. (Note that cases in which $W(x)=\infty$ for $x>x_{0}$ are also included.)

Let us introduce the decomposition $h_{i}=n_{i}+\phi_{i}$, where $n_{i} \in \mathbb{Z}$ and $-\frac{1}{2}<\phi_{i} \leqslant \frac{1}{2}$ $(i=1, \ldots, N)$. Obviously $n_{1}=0$. This transforms $\mathcal{Z}_{\mathcal{N}}(\beta)$ into

$\mathcal{Z}_{N}(\beta)=\int_{\left[-\frac{1}{2}, \frac{1}{2}\right]^{N}} \mathrm{~d} \phi \prod_{i=1}^{N} \mathrm{e}^{-\beta B\left(\phi_{i}\right)} \sum_{\mathbf{n} \in\{0\} \times \mathbb{Z}^{N-1}} \prod_{i=1}^{N} \exp \left\{-\beta W\left(n_{i-1}-n_{i}+\phi_{i-1}-\phi_{i}\right)\right\}$

where $\phi \equiv\left(\phi_{1}, \ldots, \phi_{N}\right), \mathbf{n} \equiv\left(n_{1}, \ldots, n_{N}\right)$ and, of course, $n_{0}=n_{N}$ and $\phi_{0}=\phi_{N}$. The Fourier series

$$
V(\beta, \phi, \theta) \equiv \sum_{n=-\infty}^{\infty} \mathrm{e}^{-\beta W(n+\phi)} \mathrm{e}^{-\mathrm{i} n \theta}
$$

defines a $2 \pi$-periodic function of $\theta$. Condition (4) makes the series (6) converge uniformly in $\theta$ for every $\phi \in \mathbb{R}$ and $\operatorname{Re} \beta>0$; hence $V$ is a continuous function of $\theta$. Also, if $W(x)>0$ for all $x$ sufficiently large, the series converges uniformly in compacts of $\operatorname{Re} \beta>0$, so by the analytic convergence theorem $V$ is holomorphic in $\operatorname{Re} \beta>0$.

We can now introduce the formula for the coefficients of (6),

$$
\mathrm{e}^{-\beta W(n+\phi)}=\frac{1}{2 \pi} \int_{-\pi}^{\pi} \mathrm{d} \theta V(\beta, \phi, \theta) \mathrm{e}^{\mathrm{i} n \theta}
$$

into equation (5) and make use of the identity $\sum_{n \in \mathbb{Z}} \mathrm{e}^{\mathrm{i} n x}=2 \pi \sum_{k \in \mathbb{Z}} \delta(x-2 k \pi)$ to get

$$
\mathcal{Z}_{N}(\beta)=\int_{\left[-\frac{1}{2}, \frac{1}{2}\right]^{N}} \mathrm{~d} \phi \prod_{i=1}^{N} \mathrm{e}^{-\beta B\left(\phi_{i}\right)} \frac{1}{2 \pi} \int_{-\pi}^{\pi} \mathrm{d} \theta \prod_{i=1}^{N} V\left(\beta, \phi_{i-1}-\phi_{i}, \theta\right) .
$$

If we define the integral operator

$$
\begin{aligned}
& \mathrm{T}_{\beta, \theta} f(\phi) \equiv \int_{-1 / 2}^{1 / 2} \mathrm{~d} \phi^{\prime} \mathcal{T}_{\beta, \theta}\left(\phi, \phi^{\prime}\right) f\left(\phi^{\prime}\right) \\
& \mathcal{T}_{\beta, \theta}\left(\phi, \phi^{\prime}\right) \equiv V\left(\beta, \phi-\phi^{\prime}, \theta\right) \exp \left\{-\frac{\beta}{2}\left[B(\phi)+B\left(\phi^{\prime}\right)\right]\right\}
\end{aligned}
$$


the partition function (8) adopts the simple form

$$
\mathcal{Z}_{N}(\beta)=\frac{1}{2 \pi} \int_{-\pi}^{\pi} \mathrm{d} \theta \operatorname{Tr}\left(\operatorname{T}_{\beta, \theta}\right)^{N}
$$

Except for the average in $\theta, \mathrm{T}_{\beta, \theta}$ is a transfer operator for this model.

According to the definition (6), $V(\beta,-\phi, \theta)=V(\beta, \phi, \theta)^{*}$; on the other hand,

$$
\int_{-1 / 2}^{1 / 2} \mathrm{~d} \phi \int_{-1 / 2}^{1 / 2} \mathrm{~d} \phi^{\prime}\left|\mathcal{T}_{\beta, \theta}\left(\phi, \phi^{\prime}\right)\right|^{2}<\infty
$$

so for every $-\pi<\theta<\pi$ and $\beta>0, \mathrm{~T}_{\beta, \theta}$ is a Hilbert-Schmidt operator in $L^{2}\left(\left[-\frac{1}{2}, \frac{1}{2}\right]\right)$ (hence compact and Hermitian). The whole spectrum of a compact, Hermitian operator (Young 1988) consists of a finite or infinite sequence of real, isolated eigenvalues, $\lambda_{n}$. If infinite, the sequence tends to zero (which may or may not be itself an eigenvalue). Besides, because of (12), a Hilbert-Schmidt operator also satisfies $\sum_{n} \lambda_{n}^{2}<\infty$. Consequently,

$$
\operatorname{Tr}\left(\mathrm{T}_{\beta, \theta}\right)^{N}=\sum_{n \geqslant 1}\left[\lambda_{n}(\beta, \theta)\right]^{N}=m(\beta, \theta)\left[\lambda_{\max }(\beta, \theta)\right]^{N}[1+o(1)] \quad \text { as } \quad N \rightarrow \infty
$$

$m(\beta, \theta)$ being the (finite) multiplicity of the largest eigenvalue $\lambda_{\max }(\beta, \theta)$ (which is then necessarily positive). Note that the above series converges for any $N \geqslant 2$.

If we now apply Laplace's method (de Bruijn 1981),

$$
-f(\beta) \equiv \lim _{N \rightarrow \infty} \frac{1}{N} \ln \mathcal{Z}_{N}(\beta)=\max _{-\pi \leqslant \theta \leqslant \pi} \ln \left[\lambda_{\max }(\beta, \theta)\right] .
$$

Now, $0<\operatorname{Tr}\left(\mathrm{T}_{\beta, \theta}\right)^{N}=\left|\operatorname{Tr}\left(\mathrm{T}_{\beta, \theta}\right)^{N}\right| \leqslant \operatorname{Tr}\left|\mathrm{T}_{\beta, \theta}\right|^{N} \leqslant \operatorname{Tr}\left(\mathrm{T}_{\beta, 0}\right)^{N}$, where $\left|\mathrm{T}_{\beta, \theta}\right|$ denotes the integral operator with kernel the absolute value of the kernel (10), and the last inequality follows from the definition (6). This implies that $\lambda_{\max }(\beta, \theta)$ attains its maximum value at $\theta=0$. But $\mathrm{T}_{\beta, 0}$ satisfies the hypothesis of the Jentzsch-Perron theorem for positive operators in Banach lattices (Meyer-Nieberg 1991), so $\lambda_{\max }(\beta, 0)$ has multiplicity 1 for any $\beta>0$. This being so, the holomorphy of the kernel in $\operatorname{Re} \beta>0$ implies that of $\lambda_{\max }(\beta, 0)$ (Kato 1995) and hence of $f(\beta)$ in (14).

\section{Conclusions}

We have proved that the free energy of a class of 1D models for growth of crystalline thin films is analytical for any finite temperature. This mathematical result translates into the physical realm as a strict prohibition of phase transitions in this class of models. To our knowledge, this is the first time that such a theorem is rigorously shown true for these models. Note, however, that the analyticity of the free energy does not exclude the existence of more or less sharp (dynamical) crossovers in the model behaviour: all we prove here is that these systems cannot have a true, thermodynamic phase transition.

A question that the result we are reporting in this paper immediately gives rise to is whether or not the result can be generalized to (a) any one-dimensional model, or at least to (b) any growth model. The answer to (a) is no, as there are well-known and old counterexamples (e.g., Nagle 1968, Kittel 1969; note that these models include forbidden, infinite energy configurations which render their transfer matrices reducible and imply that the PerronFrobenius (Horn and Johnson 1985) theorem does not apply to them, making possible their phase transitions) and more recent ones (Dauxois and Peyrard 1995, Dauxois et al 2001; these models do not have infinite energy configurations, as in our problem); restricting ourselves to growth models, the answer to (b) is also no (Yeomans 1992, Cuesta et al 2001). However, the theorem we are reporting here can be extended to include a much wider class of models than 
those discussed here. Such a generalization will be the subject of a forthcoming publication (Cuesta and Sánchez 2001).

\section{Acknowledgments}

The authors gratefully acknowledge Saúl Ares and Raúl Toral for highly valuable discussions, and Antonio García for a critical reading of the manuscript. AS is also grateful to Raúl Toral for his very kind hospitality at IMEDEA (Palma de Mallorca, Spain) where part of this work was done, and to the Facultat de Ciéncies of the Universitat de les Illes Balears for support of his stay at Palma de Mallorca. This work was supported by the Dirección de Ciencia y Tecnología of the Ministerio de Ciencia y Tecnología of Spain, through projects BFM2000-0004 (JAC) and BFM2000-0006 (AS).

\section{References}

Chui S T and Weeks J D 1978 Phys. Rev. Lett. 40733

Cuesta J A and Sánchez A 2001 unpublished

Cuesta J A, Sánchez A, Ares S and Toral R 2001 unpublished

Dauxois T and Peyrard M 1995 Phys. Rev. E 514027

Dauxois T, Theodorakopoulos N and Peyrard M 2001 Preprint cond-mat/0105341

de Bruijn N G 1981 Asymptotic Methods in Analysis (New York: Dover) ch 4

Currie J F, Fogel M B and Palmer F L 1977 Phys. Rev. A 16796

Gupta N and Sutherland B 1976 Phys. Rev. A 141790

Guyer R A and Miller M D 1978 Phys. Rev. A 171205

Horn R A and Johnson C R 1985 Matrix Analysis (Cambridge: Cambridge University Press) p 500ff

Kato T 1995 Perturbation Theory for Linear Operators (Berlin: Springer) p 368

Kittel C 1969 Am. J. Phys. 37917

Lieb E H and Mattis D C 1966 Mathematical Physics in One Dimension (New York: Academic) p 3

Meyer-Nieberg P 1991 Banach Lattices (Berlin: Springer) p 273

Nagle J F 1968 Am. J. Phys. 361114

Ruelle D 1989 Statistical Mechanics: Rigorous Results (Reading: Addison-Wesley) p 134

Schneider T and Stoll E 1980 Phys. Rev. B 225317

Tsuzuki T and Sasaki K 1988 Prog. Theor. Phys. Suppl. 9473

van Hove L 1950 Physica 16137 (reprinted in Lieb and Mattis 1966 p 28)

Weeks J D and Gilmer G H 1979 Adv. Chem. Phys. 40157

Yeomans J M 1992 Statistical Mechanics of Phase Transitions (Oxford: Oxford University Press) p 77

Young N 1988 An Introduction to Hilbert Space (Cambridge: Cambridge University Press) ch 8 